Pacific Journal of Mathematics

MINIMAL NONCOMMUTATIVE VARIETIES AND PONE 


\title{
MINIMAL NONCOMMUTATIVE VARIETIES AND POWER VARIETIES
}

\author{
Stuart W. Margolis and Jean-Eric Pin
}

\begin{abstract}
A variety of finite monoids is a class of finite monoids closed under taking submonoids, quotients and finite direct products. A language $L$ is a subset of a finitely generated free monoid. The variety theorem of Eilenberg sets up a one to one correspondence between varieties of finite monoids and classes of languages called, appropriately, varieties of languages. Recent work in variety theory has been concerned with relating operations on varieties of languages to operations on the corresponding variety of monoids and vice versa. For example, passing from a variety $\mathrm{V}$ of monoids to the variety $\mathrm{PV}$ generated by the power monoids of members of $\mathbf{V}$ corresponds to the operations of inverse substitution and literal morphism on varieties of languages. Recall that the power monoid of a monoid $M$ is the power set $P M$ with the usual multiplication of subsets. In this paper we consider iterating the operation which assigns $P V$ to $V$. We show in particular that $P^{3} V=P^{4} V$ for any variety $V$ and that the exponent 3 is the best possible. In fact if $V$ contains a non-commutative monoid, then $P^{3} V$ is the variety of all finite monoids.

The proof of this theorem depends upon a classification of the minimal noncommutative varieties. A variety is minimal noncommutative if all its proper subvarieties contain only commutative monoids. We show that such a variety is either generated by a noncommutative metabelian group or by the syntactic monoid of one of the languages $A^{*} a, a A^{*}$ or $\{a b\}$ over the alphabet $A=\{a, b\}$.
\end{abstract}

Let $L$ be a language over the finite alphabet $A$. The syntactic monoid $M(L)$ of $L$ is the quotient of the free monoid $A^{*}$ by the largest congruence such that $L$ is a union of classes. $L$ is said to be recognizable if $M(L)$ is finite. Syntactic monoids have been used extensively to classify recognizable languages. For example, a language is rational if it can be obtained from the letters of the alphabet by applying the operations union, concatenation and star (or submonoid generated) a finite number of times. Kleene's theorem states that a language $L$ is rational if and only if $L$ is recognizable. A language is star-free if $L$ can be obtained from the letters of $A$ by applying the operations union, complement and concatenation a finite number of times. Schützenberger's theorem says that $L$ is star-free if and only if $M(L)$ is a finite aperiodic monoid. That is every subgroup in $M(L)$ is trivial. These two important results are special cases of Eilenberg's variety theorem. We refer the reader to the books by Eilenberg [1] and Lallement [2] for details and many more examples. 
The study of the operation $\mathbf{V} \rightarrow \mathbf{P V}$ and its relationship with language theory appeared first in the work of Reutenauer [10] and Straubing [11]. In particular, Straubing asked whether $\mathbf{P}^{2} \mathbf{V}=\mathbf{P}^{3} \mathbf{V}$ for every variety $\mathbf{V}$ and proved that this is true if $\mathbf{V}$ is a variety of commutative monoids, a result obtained independently by Perrot in [5]. In [6] it was proved that $\mathbf{P}^{4} \mathbf{V}$ is the variety of all finite monoids, if $\mathbf{V}$ contains a noncommutative monoid and thus $\mathbf{P}^{\mathbf{4}} \mathbf{V}=\mathbf{P}^{\mathbf{5}} \mathbf{V}$ for every variety $\mathbf{V}$. An example was also given of a variety $\mathbf{V}$ for which $\mathbf{P}^{2} \mathbf{V} \neq \mathbf{P}^{3} \mathbf{V}$. Thus the question remained opened as to whether $\mathbf{P}^{3} \mathbf{V}=\mathbf{P}^{4} \mathbf{V}$ for all varieties $\mathbf{V}$. Our main result settles this question.

In $\$ 2$ we completely classify the minimal noncommutative varieties by showing that such a variety is either generated by a noncommutative metabelian group or by the syntactic monoid of one of the languages $A^{*} a$, $a A^{*}$ or $\{a b\}$ over the alphabet $A=\{a, b\}$. After reviewing the power variety operation in $\S 3$, we prove the result on the hierarchy $\mathbf{P}^{\mathbf{n}} \mathbf{V}$ in $\S 4$. We will see that the variety $\mathbf{J}$ of $q$ trivial monoids plays an important role.

1. Some preliminaries. In this paper all monoids are finite, except in the case of a free monoid $A^{*}$ over a finite alphabet $A$. All undefined notions can be found in [1] or [2]. In particular, we assume knowledge of the Green relations, the syntactic monoid $M(L)$ of a language $L$, and the elementary properties of varieties of finite monoids and varieties of languages.

We will say that the monoid $M$ recognizes a language $L \subseteq A^{*}$ if there exists a morphism $\phi: A^{*} \rightarrow M$ and a subset $P$ of $M$ such that $L=P \phi^{-1}$. It is well known that $M$ recognizes $L$ if and only if $M(L)$ divides $M$ (written $M(L)<M)$.

We now define some monoids which will play an important role in what follows. Recall that $U_{2}$ is the monoid consisting of two right zeroes and an identity. $U_{2}^{r}$ will denote the reverse of $U_{2}$. For each $n \geq 0$, let $\mathbf{n}=\{0, \ldots, n-1\}$. Let $B A_{n}$ denote the monoid consisting of all partial functions $f: \mathbf{n} \rightarrow \mathbf{n}$ with the property that $\operatorname{card}\left(\mathbf{n} f^{-1}\right) \leq 1$, together with the identity function on $\mathbf{n} . B A_{n}$ is called the aperiodic Brandt monoid of size $n$. Finally we let $N$ be the syntactic monoid of the language $\{a b\}$ over the alphabet $A=\{a, b\}$. It is easy to see that the minimal automaton of $\{a b\}$ is given by:

$$
\rightarrow \text { (1) } \stackrel{a}{\rightarrow} \text { (1) } \stackrel{b}{\rightarrow} \text { (2) } \rightarrow
$$

and thus $N$ divides $B A_{3}$. 
The importance of the monoid $N$ is given by the following result of Pin. We will say that the language $L$ is noncommutative if $M(L)$ is a noncommutative monoid. A word $w \in A^{*}$ is multilinear if every $a \in A$ occurs at most once in $w$.

Proposition 1.1. Let $\mathbf{V}$ be a variety and let $\mathfrak{V}$ be the corresponding variety of languages. The following conditions are equivalent.

(1) $N \in \mathbf{V}$

(2) $\mathcal{T}$ contains a finite noncommutative language.

(3) $A^{*} \mathcal{V}$ contains all the languages $\{w\}$ where $w \in A^{*}$ is multilinear.

Proof. See [6].

The variety $\mathbf{J}$ consists of all monoids $M$ such that for all $m, n \in M$, $M m M=M n M$ implies $m=n$. A language $L$ is piecewise testable if $L$ is contained in the Boolean closure of the languages $A^{*} a_{1} A^{*} a_{2} \cdots a_{n} A^{*}$ where $a_{\imath} \in A i=1, \ldots, n$.

Simon's Theorem. A language $L$ is piecewise testable if and only if $M(L) \in \mathbf{J}$.

2. Minimal noncommutative varieties. Recall that a group $G$ is metabelian if its derived group $D G$ is abelian.

THEOREM 2.1. Let $\mathbf{V}$ be a variety of monoids. Then $\mathbf{V}$ is noncommutative if and only if $\mathbf{V}$ contains a noncommutative metabelian group or one of the monoids $U_{2}, U_{2}^{r}$ or $N$.

Recall that $N$ is the syntactic monoid of the language $\{a b\}$ over the alphabet $\{a, b\}$.

The proof of Theorem 2.1 will follow from a sequence of lemmata. The following lemma is of independent interest.

LEMMA 2.2. Let $G$ be a commutative group which is a 1 -class of a semigroup $S$ and let $u, v \in S$. If $G$ contains any two of $u, v, u v, v u$, then $u v=v u$ is an element of $G$.

Proof. Let $e$ be the identity of $G$. Clearly the result holds if $u, v \in G$. Assume that $u, u v \in G$. Then $u v e \in G$ so that $v e g e$. Since $G$ is a $\mathscr{D}$-class it follows that $v e \in G$. Therefore

$$
u v=u(v e)=(v e) u=v(e u)=v u .
$$


If $u v, v u \in G$, then euve, evue $\in G$. As above it follows that $u e, v e$, $e u, e v \in G$. Therefore

$$
u v=(e u)(v e)=(v e)(e u)=(\text { eve })(\text { eue })=e v u e=v u .
$$

The other cases follow by duality.

Proof of Theorem 2.1. If $\mathbf{V}$ contains a noncommutative group $G$, then it contains $(\mathbf{G})$, the variety generated by $G$. It follows from a theorem of $S$. Oates [4, Theorem 21.4], that (G) contains a noncommutative metabelian group. We remark that the proof is given for varieties in the sense of Birkhoff, but that the proof works also for $M$-varieties. The proof follows from a result of $\mathrm{O}$. J. Schmidt which shows that a finite group, all of whose subgroups are abelian, is metabelian [9, pg. 721]. Assume every group in $\mathbf{V}$ is commutative and that $U_{2}, U_{2}^{r}$ and $N$ are not in $\mathbf{V}$. We will show that $\mathbf{V}$ is a commutative variety. We first prove some properties of members of $\mathbf{V}$. Let $T$ be in $\mathbf{V}$.

\section{LEMMA 2.3. Every regular $\mathscr{D}$-class of $T$ is a commutative group.}

Proof. Let $D$ be a regular $\mathscr{Q}$-class of $T$. Since $U_{2}$ and $U_{2}^{r}$ are not in $\mathbf{V}$,

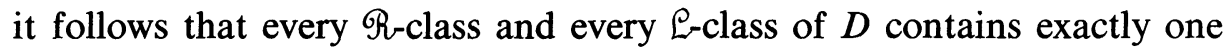
idempotent. Since $N$ is not in $\mathbf{V}, B A_{2}$ is not in $\mathbf{V}$, since $N<B A_{3}<B A_{2} \times$ $B A_{2}$. It follows that $D$ is a group. But every group in $\mathbf{V}$ is commutative and the result follows.

LEMMA 2.4. Let $u, v \in T$. If $u v \Re u(v u L u)$, then $u v=v u$.

Proof. We prove $u v \Re u$ implies $u v=v u$. The other case is dual. If $u$ is regular, then by Lemma 2.3 the $\mathscr{D}$-class of $u$ is a commutative group $G$. Since $u, u v \in G, u v=v u$ by Lemma 2.2. Assume that $u$ nonregular and $u v \neq v u$. Let $I$ be the ideal of $T$ defined by $I=\left\{x \in T, x<_{g} u\right\}$, and let $T^{\prime}=T / I$. Let $R$ be the submonoid of $T^{\prime} \times T^{\prime}$ generated by $x=(u, v)$ and $y=(1, u)$ and let $J=\left(T^{\prime} \times 0\right) \cup\left(0 \times T^{\prime}\right)$. We claim that $N$ divides $R /(J \cap R)=R^{\prime}$. Indeed, since $u$ is nonregular, $u v u, u^{2} \in I$ and therefore $x^{2}=y^{2}=x y x=y x y=0$ in $R^{\prime}$. Moreover $x y \neq y x$ and $y x \neq 0$. Therefore $N$ is isomorphic to the quotient of $R^{\prime}$ by the ideal generated by $x y$. Thus the claim holds and $N \in \mathbf{V}$, a contradiction.

LEMMA 2.5. Green's relations $\mathcal{H}$ and $\mathcal{G}$ coincide in $T$. 
Proof. Suppose that $u \Re u v$ for some $u, v$ in $T$. Then $u v=v u$ by Lemma 2.4 and therefore $u \varrho v u=u v$. Thus $u \mathcal{H} u v$. This proves that $\mathcal{H}=\mathscr{R}$. Dually $\mathcal{H}=\mathcal{L}$ and thus $\mathcal{H}=\mathscr{G}$.

Now, assume that $\mathbf{V}$ contains a noncommutative monoid $S$. Let $a, b$ in $S$ be such that $a b \neq b a$ and let $M$ be the submonoid of $S$ generated by $a$ and $b$. Considering $\{a, b\}$ as an alphabet, define $\eta:\{a, b\}^{*} \rightarrow M$ by $a \eta=a$ and $b \eta=b$.

LEMMA 2.6. $a b$ is not $q$ related to ba in $M$.

Proof. By Lemma 2.5 it is sufficient to prove that $a b$ is not $\mathcal{K}$ related to $b a$ in $M$. Assume $a b \mathcal{F} b a$. Then it is well known that there exists a group $G$ in $M$ with identity $e$, and $g \in G$ such that

$$
a b g=b a \text { and } b a g^{-1}=a b .
$$

Since $a b \neq b a, g \neq 1$. Therefore there exists $u \in\{a, b\}^{+}$, such that $u \eta=e$. If $a b(b a)$ is a factor of $u$, then $a b g b a g e$ since $a b e=a b$. Therefore $a b, b a \in G$ and $a b=b a$ by Lemma 2.2.

We may assume then that $u=a^{p}$ (or $b^{p}$ ) for some $p>0$.

It follows that

$$
a b a^{p}=a b \text { and } \quad b a a^{p}=b a
$$

Consequently,

$$
a b \Re a b a \Re a b a a^{p-1}
$$

and by Lemma 2.4 we have

$$
a b=(a b a) a^{p-1}=a^{p-1}(a b a)=a^{p}(b a)=b a
$$

a contradiction.

LEMMA 2.7. If $(a b) \eta^{-1}$ is infinite, then $a b \leq_{g} b a$ in $M$.

Proof. If $b a$ is a factor of some word $w \in(a b) \eta^{-1}$, then clearly $a b \leq_{g} b a$. Therefore, we may suppose that $(a b) \eta^{-1} \subseteq a^{*} b^{*}$. Choose an integer $p>0$, such that both $a^{p}$ and $b^{p}$ are idempotents. Since $(a b) \eta^{-1}$ is infinite, there exists $a^{n} b^{m} \in(a b) \eta^{-1}$ such that $n \geq p$ or $m \geq p$. Then

$$
a b=a^{n} b^{m}=a^{n+p} b^{m}=a^{p} a b .
$$

Therefore, Lemma 2.4 implies that

$$
a^{p} a b=a b a^{p}=a b, \text { so that } a b \leq_{g} b a \text { in } M .
$$


We can now complete the proof. Since $a b$ is not $q$ related to $b a$ by Lemma 2.6 , it follows that either $(a b) \eta^{-1}$ or $(b a) \eta^{-1}$ is finite by Lemma 2.7. Therefore $M$ recognizes a finite noncommutative language and $N \in \mathbf{V}$ by Proposition 1.1. This is a contradiction and $\mathbf{V}$ is a commutative variety.

We remark that a similar result holds for varieties of semigroups. Let $B(1,2)(B(2,1))$ be the two element right zero (left zero) semigroup.

THEOREM 2.8. Let $\mathbf{V}$ be a variety of semigroups. Then $\mathbf{V}$ is noncommutative if and only if $\mathbf{V}$ contains a noncommutative metablian group or one of the semigroups $B(1,2), B(2,1)$ or the syntactic semigroup of $\{a b\}$ over the alphabet $\{a, b\}$.

Proof. We need only adapt the proof of Theorem 2.1. The only difficulty is in the proof of Lemma 2.4. It suffices to let $x=(h, v)$ and $y=(u v, u)$ where $h$ is such that $u v h=u$. The rest of the proof is identical.

We close with two important corollaries.

Corollary 2.9. Let $\mathbf{V}$ be a noncommutative variety and let $A$ be an alphabet containing at least two distinct letters $a$ and $b$. Then $A^{*} \mathbf{V}$ contains either a language recognized by a noncommuative group or one of the languages $A^{*} a, a A^{*}$, or $\{a b\}$.

Proof. Since $U_{2}, U_{2}^{r}$ and $N$ are the syntactic monoid of $A^{*} a, a A^{*}$ and $\{a b\}$ respectively, the result follows.

COROllary 2.10. Let $\mathbf{V}$ be a variety of monoids. Then $\mathbf{V}$ is commutative iff every aperiodic monoid and every group in $\mathbf{V}$ are commuative.

Proof. This follows from the fact that $U_{2}, U_{2}^{r}$ and $N$ are noncommutative aperiodic monoids.

3. A review of the operation $\mathbf{V} \rightarrow \mathbf{P V}$. In this section we review the power variety construction and the corresponding operation on *-varieties. In the next section we will use these results and the results of $\$ 2$ to show that $\mathbf{P}^{\mathbf{3}} \mathbf{V}$ is the variety of all monoids if $\mathbf{V}$ is noncommutative.

If $M$ is a monoid, then $P M$ will denote the power monoid of $M$ with the usual multiplication of subsets. If $\mathbf{V}$ is a variety, then $\mathbf{P V}$ is the variety generated by $\{P M \mid M \in \mathrm{V}\}$.

The operation $\mathbf{V} \rightarrow \mathbf{P V}$ on varieties of monoids corresponds to two important operations on varieties of languages. Let $A$ and $B$ be alphabets. 
A morphism $\phi: B^{*} \rightarrow A^{*}$ is literal if $B \phi \subseteq A$. A morphism $\sigma: A^{*} \rightarrow P B^{*}$ will be called a substitution. Let $\sigma: A^{*} \rightarrow P B^{*}$ be a substitution. If $L \in P B^{*}$, let $L \sigma^{-1}=\left\{w \in A^{*} \mid w \sigma \cap L \neq \varnothing\right\}$. Thus we treat $\sigma$ as a relation $\sigma: A^{*} \rightarrow B^{*}$ and take inverse images with respect to this relation.

If $\mathcal{V}$ is a *-variety of languages, let $A^{*} \Lambda^{\mathcal{V}}$ be the Boolean algebra generated by the languages of the form $L \phi$ where $L \in B^{*} \mathcal{V}$ for some alphabet $B$ and $\phi: B^{*} \rightarrow A^{*}$ is a literal morphism. Let $A^{*} \Sigma \mathcal{V}$ be the Boolean algebra generated by languages of the form $L \sigma^{-1}$ where $L \in B^{*} \mathcal{V}$ and $\sigma: A^{*} \rightarrow P B^{*}$ is a substitution.

The following propositions summarize the work of Pin [6], Reutenauer [9], and Straubing [11].

Proposition 3.1. Let $\mathbf{V}$ be a variety of monoids and let $\mathfrak{V}$ be the corresponding ${ }^{*}$-variety. Then $\Lambda \mathcal{V}=\Sigma \mathcal{V}$ are ${ }^{*}$-varieties which correspond to PV.

Proposition 3.2. Let $\mathbf{V}$ be a variety of monoids and let $\mathcal{V}$ be the corresponding variety of languages. The following conditions are equivalent:

(1) $\mathbf{V}=\mathbf{P V}$.

(2) $\mathcal{V}=\Lambda \mathcal{V}$.

(3) $\mathfrak{V}=\Sigma \mathcal{V}$.

4. The hierarchy $\mathbf{P}^{n} \mathbf{V}$. Let $\mathbf{V}$ be a variety and for each $n \geq 0$, let $\mathbf{P}^{\mathbf{n}+\mathbf{1}} \mathbf{V}=\mathbf{P}\left(\mathbf{P}^{\mathbf{n}} \mathbf{V}\right)$. We then have the hierarchy $\mathbf{V} \subseteq \mathbf{P V} \subseteq \mathbf{P}^{\mathbf{2}} \mathbf{V} \ldots$. In [11] Straubing asked whether this hierarchy is infinite for some variety $\mathbf{V}$. $\mathrm{He}$ conjectured that $\mathbf{P}^{2} \mathbf{V}=\mathbf{P}^{3} \mathbf{V}$ for any variety $\mathbf{V}$ and proved the following result, obtained independently by Perrot in [5].

Proposition 4.1. Let $\mathbf{V}$ be a commutative variety. Then $\mathbf{P}^{2} \mathbf{V}=\mathbf{P}^{3} \mathbf{V}$. Furthermore, if $\mathbf{V}$ contains a nontrivial monoid, then $\mathbf{P V}=\mathbf{P}^{2} \mathbf{V}$ is the variety of commutative monoids all of whose subgroups are in $\mathbf{V}$.

In [6] Pin proved the following results.

Proposition 4.2. If $\mathbf{V}$ is any noncommutative variety, then $\mathbf{P}^{4} \mathbf{V}$ is the variety of all finite monoids.

COROllary 4.3. $\mathbf{P}^{4} \mathbf{V}=\mathbf{P}^{5} \mathbf{V}$ for any variety $\mathbf{V}$.

Proposition 4.4. Let $\mathbf{R}_{\mathbf{1}}$ be the variety of $R$-trivial idempotent monoids. Then the varieties $\mathbf{R}_{\mathbf{1}}, \mathbf{P R}_{\mathbf{1}}, \mathbf{P}^{\mathbf{2}} \mathbf{R}_{\mathbf{1}}, \mathbf{P}^{\mathbf{3}} \mathbf{R}_{\mathbf{1}}$ are distinct. 
We now begin the proof that $\mathbf{P}^{3} \mathbf{V}=\mathbf{M}$ for any noncommutative variety $\mathbf{V}$.

Proposition 4.5. Let $\mathbf{V}$ be the variety generated by $N$. Then $\mathbf{P V}=\mathbf{J}$.

Proof. It is shown in [6] that if Nil is the variety generated by monoids $M$ such that $M-\{1\}$ is a nilpotent semigroup, then PNil $\subseteq \mathbf{J}$. In particu$\operatorname{lar} \mathbf{P V} \subseteq \mathbf{J}$.

Conversely, let $A$ be a finite alphabet and let $\mathscr{V}$ be the *-variety corresponding to V. It suffices by Simon's theorem and Proposition 3.1 to show that $L=A^{*} a_{1} A^{*} a_{2} \cdots a_{n} A^{*} \in A^{*} \Sigma \mathcal{V}$ for $a_{i} \in A, i=1, \ldots, n$.

Let $B=\left\{b_{1}, \ldots, b_{n}\right\}$ be an alphabet with $n$ letters. Consider the substitution $\sigma: A^{*} \rightarrow P B^{*}$ defined by $a \sigma=\{1\} \cup\left\{b_{i} \mid a=a_{i}\right\}$. But $\left\{b_{1}\right.$ $\left.\cdots b_{n}\right\} \in B^{*} \mathscr{V}$ by Proposition 1.1 and an easy calculation shows that $L=\left\{b_{1} \cdots b_{n}\right\} \sigma^{-1} \in A^{*} \Sigma \mathscr{V}$, as desired.

COROLlaRY 4.6. If $\mathbf{V}$ is a variety which recognizes a finite noncommutative language, then $\mathbf{J} \subseteq \mathbf{P V}$.

Proof. Follows from Proposition 1.1 and 4.5.

The following is a result of Pin, Straubing, and Therien [8].

Proposition 4.7. The variety generated by $U_{2}^{r}\left(U_{2}\right)$ is equal to the variety $\mathbf{R}_{1}\left(\mathbf{R}_{1}^{\mathbf{r}}\right)$.

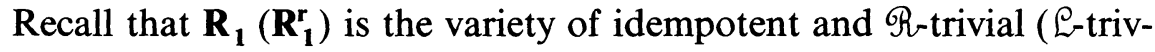
ial) monoids.

Proposition 4.8. The varieites $\mathbf{P R}_{\mathbf{1}}$ and $\mathbf{P R}_{\mathbf{1}}^{\mathbf{r}}$ contain $\mathbf{J}$.

Proof. We treat the case of $\mathbf{V}=\mathbf{R}_{\mathbf{1}}$. The case of $\mathbf{R}_{\mathbf{1}}$ follows by duality. It suffices by Simon's theorem and Proposition 3.1 to show that $L=$ $A^{*} a_{1} A^{*} a_{2}, \ldots, a_{n} A^{*} \in A^{*} \Lambda^{\mathscr{V}}$ for $a_{i} \in A, i=1, \ldots, n$, and any finite alphabet $A$. To this end let $B=\left\{b_{1}, \ldots, b_{n}\right\}$ be an $n$-letter alphabet and let $C=\left\{c_{1}, \ldots, c_{n}\right\}$ be a second $n$-letter alphabet disjoint from $B$. Let

$$
K=C^{*} b_{1}\left(C \cup\left\{b_{1}\right\}\right) * b_{2} \cdots \cdot\left(C \cup\left\{b_{1} \cdots \cdot b_{n-1}\right\}\right) * b_{n}(C \cup B)^{*}
$$

and define the literal morphism $\phi:(B \cup C)^{*} \rightarrow A^{*}$ by $c_{i} \phi=b_{i} \phi=a_{i}$, $1 \leq i \leq n$. Then $L=K \phi$, so we need only show that $K \in(B \cup C)^{* \mathscr{V}}$. 
An easy calculation shows that the minimal automaton of $K$ is given by:

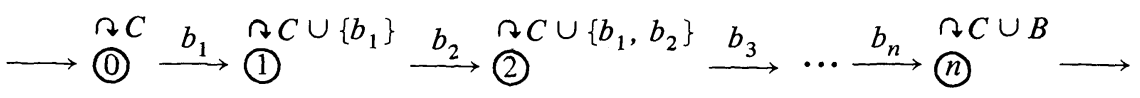

It follows that the syntactic monoid of $K$ is a submonoid of the monoid $M_{n}$ of partial functions $f_{r, s}:\{0, \ldots, n\} \rightarrow\{0, \ldots, n\}, 0 \leq r \leq s \leq n$, defined by

$$
q f_{r, s}=\left\{\begin{array}{l}
s \text { if } r \leq q \leq s \\
q \text { if } q>s \\
\text { undefined otherwise. }
\end{array}\right.
$$

The multiplication in $M_{n}$ is given by

$$
f_{r, s} f_{r^{\prime}, s^{\prime}}= \begin{cases}f_{r^{\prime}, s^{\prime}} & \text { if } s<r^{\prime}, \\ f_{r, t} & \text { if } s \geq r^{\prime} \text { and } t=\max \left\{s, s^{\prime}\right\} .\end{cases}
$$

In particular, $M_{n}$ is an idempotent monoid. Furthermore, if $f_{r, s} \Re f_{r^{\prime}, s^{\prime}}$, then $f_{r, s} f_{r^{\prime}, s^{\prime}}=f_{r^{\prime}, s^{\prime}}$ and $f_{r^{\prime}, s^{\prime}} f_{r, s}=f_{r, s}$. It follows from (1) that $f_{r, s}=f_{r^{\prime}, s^{\prime}}$

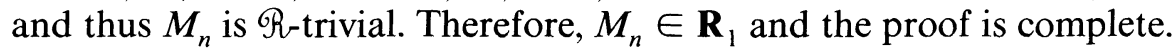

From Proposition 4.5 and 4.8 we deduce

THEOREM 4.9. If $\mathbf{V}$ is a non-commutative variety such that every group in $\mathbf{V}$ is commutative, then $\mathbf{J}$ is contained in $\mathbf{P V}$.

Proof. It follows from Theorem 2.1 that $\mathbf{V}$ contains either $U_{2}, U_{2}^{r}$ or $N$. Therefore Propositions 4.5 and 4.8 imply the result.

We now give a necessary and sufficient condition for a variety $\mathbf{V}$ to be such that $\mathbf{P V}$ is the variety $\mathbf{M}$ of all finite monoids. Recall that $B A_{2}$ is the aperiodic Brandt monoid of size 2 and that DS is the variety of monoids all of whose regular $\mathscr{Q}$-classes are subsemigroups.

Proposition 4.10. Let $\mathbf{V}$ be an M-variety. The following conditions are equivalent.

(1) $B A_{2} \in \mathbf{V}$.

(2) $\mathbf{P V}=\mathbf{M}$.

(3) $\mathbf{V}$ is not contained in $\mathbf{D S}$.

The implication (1) implies (2) was given in [6] where it was also conjectured that Proposition 4.10 was true. The proof of Proposition 4.10 appears in [3]. 
THEOREM 4.11 ([6]). If $\mathbf{V}$ contains a noncommutative group, then $\mathbf{P}^{2} \mathbf{V}=\mathbf{M}$.

In effect, it is shown that $B A_{2} \in \mathbf{P V}$ and the result follows from Proposition 4.10.

Corollary 4.12. If $\mathbf{H}$ is a variety of groups, then $\mathbf{P}^{2} \mathbf{H}=\mathbf{P}^{3} \mathbf{H}$.

This follows immediately from Proposition 4.1 and Theorem 4.11.

Proposition 4.13. $\mathbf{P}^{2} \mathbf{J}=\mathbf{M}$.

Proof. By Proposition 4.10 we need only show that PJ is not contained in DS. To this end, let $A=\{a, b, c, d\}$ and let $B=\{a, b\}$. Let $K=\{c, d\}^{*} a b\{c, d\}^{*}$. A straightforward calculation shows $M(K) \in \mathbf{J}$. Define a literal morphism $\phi: A^{*} \rightarrow B^{*}$ by $a \phi=b \phi=a, c \phi=a, d \phi=b$. Then $L=K \phi=B^{*} a^{2} B^{*}$. Therefore, $M(L) \in \mathbf{P J}$ by Proposition 3.1. Another calculation shows that $M(L)$ contains a $\mathscr{D}$-class with $4 \mathcal{F}$-classes and 3 idempotents. Thus PJ is not contained in DS, as desired.

THEOREM 4.14. If $\mathbf{V}$ is a noncommutative variety, then $\mathbf{P}^{3} \mathbf{V}$ is the variety of all finite monoids.

Proof. By Theorem 2.1 V contains either a noncommutative group or one of the monoids $U_{2}, U_{2}^{r}$ or $N$. The result now follows from Theorem 4.9, Theorem 4.11 and Proposition 4.13.

Corollary 4.15. $\mathbf{P}^{3} \mathbf{V}=\mathbf{P}^{4} \mathbf{V}$ for any variety $\mathbf{V}$.

Proof. Follows from Proposition 4.1 and Theorem 4.14.

Proposition 4.4 implies that the number 3 in Corollary 4.15 is the best possible.

If $M$ is a monoid, then $M^{n}$ denotes the direct product of $n$ copies of $M$. Let $P^{\prime}(M)$ be the monoid of nonempty subsets of $M$. If $\mathbf{V}$ is a variety, then $\mathbf{P}^{\prime} \mathbf{V}$ is the variety generated by $\left\{P^{\prime}(M) \mid M \in \mathbf{V}\right\}$. The following appear in [6].

Lemma 4.16. Let $M$ and $N$ be monoids. Then $P^{\prime}(M) \times P^{\prime}(N)$ divides $P^{\prime}(M \times N)$. 
Lemma 4.17. If $\mathbf{V}$ is a nontrivial variety, then $\mathbf{P}^{\prime} \mathbf{V}=\mathbf{P V}$.

Proposition 4.18. Let $G$ be a noncommutative group and let $M$ be a monoid. There exists an integer $n$ such that $M$ divides $P\left(P\left(G^{n}\right)\right)$.

We close with the following generalization.

Proposition 4.19. Let $N$ be a noncommutative monoid and let $M$ be a monoid. There exists an integer $n$ such that $M$ divides $P\left(P\left(P\left(N^{n}\right)\right)\right)$.

Proof. By Theorem 4.14, $\mathbf{P}^{3} \mathbf{V}=\mathbf{M}$ where $\mathbf{V}$ is the variety generated by $N$. It follows from Lemmata 4.16 and 4.17 , that if $M \in \mathbf{P}^{3} \mathbf{V}$, then $M$ divides $P\left(P\left(P\left(N^{n}\right)\right)\right)$ for some $n$ and the result follows.

\section{REFERENCES}

[1] S. Eilenberg, Automata, Languages and Machines, Vol. B, Academic Press, New York, (1976).

[2] G. Lallement, Semigroups and Combinatorial Applications, Wiley, New York, 1979.

[3] S. W. Margolis, On M-Varieties generated by power monoids, Semigroup Forum, 22 (1981), 339-354.

[4] H. Neumann, Varieties of Groups, Ergebnisse der Mathematik, Springer (1967).

[5] J. F. Perrot, Varieties of languages and operations, Theoretical Computer Science, 7 (1978), 197-210.

[6] J. E. Pin, Variétés de langages et monoïde des parties, Semigroup Forum, 20 (1980), 11-47.

[7] _ Varietes de langages et varietes de semigroupes, These d'Etat, Paris, April 1981.

[8] J. E. Pin, H. Straubing and D. Thérien, Small varieties of finite semigroups and extensions, to appear in J. Australian Math. Soc.

[9] L. Redei, Algebra, Akademische Verlagsgesellschaft 1959 and Pergamon Press Oxford 1967.

[10] C. Reutenauer, Sur les variétés de langages et de monoüdes, 4th GI Conference Lecture Notes in Computer Science No. 67, Springer 260-265.

[11] H. Straubing, Aperiodic homomorphism and the concatenation product of recognizable sets, J. Pure and Applied Algebra, 15 (1979), 319-327.

Received July 24, 1981.

UNIVERSITY OF VERMONT

BURLINGTON, VT 05450

AND

CNRS AND

PARIS UNIVERSITY VI

PARIS, France 



\section{PACIFIC JOURNAL OF MATHEMATICS}

EDITORS

Donald BabBITT (Managing Editor)

University of California

Los Angeles, CA 90024

Hugo Rossi

University of Utah

Salt Lake City, UT 84112

C. C. Moore and Arthur Ogus

University of California

Berkeley, CA 94720
J. DugunduI

Department of Mathematics

University of Southern California

Los Angeles, CA 90089-1113

R. FINN and H. SAMELSON

Stanford University

Stanford, CA 94305

\section{ASSOCIATE EDITORS}
R. ARENS
E. F. BECKENBACH
B. H. NeumanN
F. WolF
K. YosHIDA (1906-1982)

\section{SUPPORTING INSTITUTIONS}

UNIVERSITY OF ARIZONA

UNIVERSITY OF BRITISH COLUMBIA

CALIFORNIA INSTITUTE OF TECHNOLOGY

UNIVERSITY OF CALIFORNIA

MONTANA STATE UNIVERSITY

UNIVERSITY OF NEVADA. RENO

NEW MEXICO STATE UNIVERSITY

OREGON STATE UNIVERSITY
UNIVERSITY OF OREGON

UNIVERSITY OF SOUTHERN CALIFORNIA

STANFORD UNIVERSITY

UNIVERSITY OF HAWAII

UNIVERSITY OF TOKYO

UNIVERSITY OF UTAH

WASHINGTON STATE UNIVERSITY

UNIVERSITY OF WASHINGTON 


\section{Pacific Journal of Mathematics}

\section{Vol. 111, No. $1 \quad$ November, 1984}

Harald Brandenburg and Adam Stefan Mysior, For every Hausdorff

space $Y$ there exists a nontrivial Moore space on which all continuous

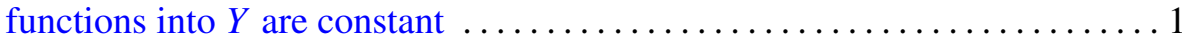

Henry Dappa, A Marcinkiewicz criterion for $L^{p}$-multipliers $\ldots \ldots \ldots \ldots \ldots 9$

P. H. Doyle, III and John Gilbert Hocking, Bijectively related spaces. I.

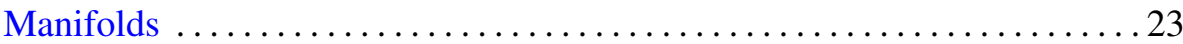

Joel Hass, Complete area minimizing minimal surfaces which are not totally

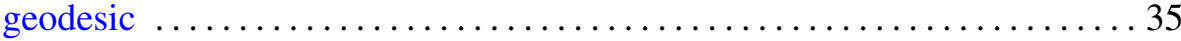

Aarno Hohti, On Ginsburg-Isbell derivatives and ranks of metric spaces .... 39

Richard Howard Hudson, Diophantine determinations of $3^{(p-1) / 8}$ and $5^{(p-1) / 4}$

A. F. Izé and A. Ventura, Asymptotic behavior of a perturbed neutral functional-differential equation related to the solution of the unperturbed

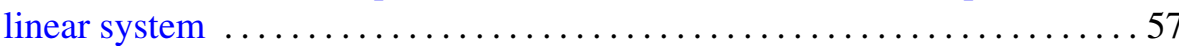

Palle E. T. Jorgensen, Spectral representations of unbounded nonlinear

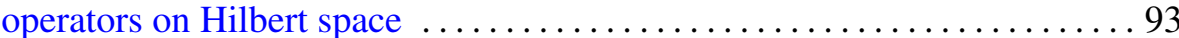

Darrell Conley Kent and Gary Douglas Richardson, Cauchy spaces with

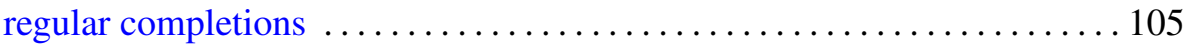

Mark Mahowald, An addendum to: "bo-resolutions" ................ 117

Stuart Wayne Margolis and Jean-Eric Pin, Minimal noncommutative varieties and power varieties

Carla Massaza and Alfio Ragusa, Some conditions on the homology groups of the Koszul complex

Vicente Miquel Molina, Some examples of Riemannian almost-product manifolds

Roderic Murufas, Inverse spectral problems for certain differential operators

Ulrich Oertel, Closed incompressible surfaces in complements of star links

Katsuro Sakai, A characterization of local equiconnectedness

William Victor Smith and Don Harrell Tucker, Weak integral convergence theorems and operator measures 